\title{
Upregulation of cathepsin D in the caudate nucleus of primates with experimental parkinsonism
}

\author{
Sowmya V Yelamanchili', Amrita Datta Chaudhuri ${ }^{1}$, Claudia T Flynn ${ }^{2}$ and Howard S Fox ${ }^{1,3^{*}}$
}

\begin{abstract}
Background: In Parkinson's disease there is progressive loss of dopamine containing neurons in the substantia nigra pars compacta. The neuronal damage is not limited to the substantia nigra but progresses to other regions of brain, leading to loss of motor control as well as cognitive abnormalities. The purpose of this study was to examine causes of progressive damage in the caudate nucleus, which plays a major role in motor coordination and cognition, in experimental Parkinson's disease.

Results: Using chronic 1-methyl-4phenyl-1,2,3,6-tetrahydropyridine treatment of rhesus monkeys to model Parkinson's disease, we found a upregulation of Cathepsin D, a lysosomal aspartic protease, in the caudate nucleus of treated monkeys. Immunofluorescence analysis of caudate nucleus brain tissue showed that the number of lysosomes increased concurrently with the increase in Cathepsin D in neurons. In vitro overexpression of Cathepsin $\mathrm{D}$ in a human neuroblastoma cell line led to a significant increase in the number of the lysosomes. Such expression also resulted in extralysosomal Cathepsin D and was accompanied by significant neuronal death associated with caspase activation. We examined apoptotic markers and found a strong correlation of Cathepsin D overexpression to apoptosis.

Conclusions: Following damage to the substantia nigra resulting in experimental Parkinson's disease, we have identified pathological changes in the caudate nucleus, a likely site of changes leading to the progression of disease. Cathepsin D, implicated in pathogenic mechanisms in other disorders, was increased, and our in vitro studies revealed its overexpression leads to cellular damage and death. This work provides important clues to the progression of Parkinson's, and provides a new target for strategies to ameliorate the progression of this disease.
\end{abstract}

Keywords: Parkinson?'?s, MPTP, striatum, caudate, neurodegeneration, cathepsin, apoptosis, nonhuman primate

\section{Background}

Parkinson's disease (PD) is the second most common neurodegenerative disease. Apart from its genetic predisposition, most cases arise sporadically and factors including drugs and toxic chemicals have been demonstrated to induce PD [1]. While medical (e.g. L-3,4-dihydroxypheylalanine, L-DOPA) and surgical (e.g. deep brain stimulation) therapies have been effective in the treatment of PD to a certain extent, nothing to date is capable of arresting disease progression.

\footnotetext{
* Correspondence: hfox@unmc.edu

'Department of Pharmacology and Experimental Neuroscience, University of Nebraska Medical Center, Omaha, NE 68198, USA

Full list of author information is available at the end of the article
}

In $\mathrm{PD}$, there is progressive loss of dopamine (DA) containing neurons in the substantia nigra (SN) pars compacta. Motor symptoms initially dominate the clinical picture, and as the disease progresses cognitive abnormalities are often evident $[1,2]$. While numerous studies examine damage and neuronal loss in the SN, other neuronal systems and brain regions are also affected [3]; and the nature of this additional neuronal damage is relatively unknown.

Understanding the dysregulation of genes and proteins involved in neuronal dysfunction and disease progression can open avenues for new therapeutic targets in $\mathrm{PD}$. One main outflow tract from the $\mathrm{SN}$ is to the striatum where the presynaptic dopaminergic terminals

\section{Ciomed Central}


originating in the SN are lost [4]. To address other PDrelated changes in the striatum, we targeted the caudate nucleus $(\mathrm{CN})$, a part of the striatum that not only receives dopaminergic input from the $\mathrm{SN}$ as well as the ventral tegmental area but also glutaminergic projections from prefrontal associational and anterior cingulate limbic areas. Similar to other regions of the striatum, the $\mathrm{CN}$ is involved in motor function and also has an important role in cognition. Imaging studies have linked abnormalities in the striatum to altered cognitive executive functions in those with PD [5-9].

A common cord in many aspects of neuronal survival is the lysosomal pathways [10]. The lysosomal proteases, which play a key role in intracellular proteolysis and in extracellular remodeling [11], are important in maintaining homeostasis by exerting degradation and regulatory functions. Lysosomal dysfunctions have been specifically associated with degenerative phenomenon [12] as well as with age-related neurological diseases [13]. Among the most powerful hydrolytic enzymes in the lysosomes are the cathepsins. Cathepsin D (Cat D) is the major intracellular aspartic protease and is present in relatively high concentrations within the lysosomes. Previous studies in a variety of cell systems such as endothelial cells, T-lymphocytes and fibroblasts have shown that increases in Cat D leads to apoptosis (reviewed in [14]). Cat D upregulation has been associated with neurodegenerative disorders including Alzheimer's disease (AD) and its presence extracellularly in senile plaques was clearly noted [15]. The upregulation of Cat D was shown to occur at an early stage in experimental models of AD leading to slow apoptosis of neurons [16]. Therefore, we questioned whether deregulation of lysosomal Cat $\mathrm{D}$ is involved in the progressive neuronal damage as seen in PD, focusing on the $\mathrm{CN}$.

We hypothesized that Cat D can act as a molecular trigger for neuronal damage in $\mathrm{CN}$. Using a chronic 1methyl-4phenyl-1,2,3,6-tetrahydropyridine (MPTP) treated rhesus monkey model, we assessed the expression level of Cat D in CN followed by immunohistochemical analysis on brain sections. Next, we performed in vitro overexpression of Cat D and subsequently followed the results by functional and imaging studies to confirm our hypothesis.

\section{Results}

\section{MPTP-treatment of monkeys}

Monkeys are sensitive to the PD-like effects of MPTP and have a good correspondence of brain function, neurochemistry, and neuroanatomy to humans. In monkeys, both acute and chronic dosing protocols can lead to a PD-like disease. While the acute effects of high dose MPTP in experimental animals can be dramatic, the acute toxicity differs from the course of PD. In addition, functional recovery can occur in a variety of dosing protocols.

Chronic MPTP dosing cannot only lead to stable motor deficits but also cognitive abnormalities as seen in PD. In order to better mimic the chronic nature of PD, we utilized a repeated low-dose protocol, in which animals received a dose of $0.3-0.4 \mathrm{mg} / \mathrm{kg}$ MPTP on two consecutive days, followed by assessment of stable effects of the treatment at 3-4 weeks post-treatment. Animals then received repeat dosing in order to achieve a state of stable, mild functional deficits. Following treatment, animals displayed behavioral changes (less grooming and social interaction when housed with another monkey and aggressiveness to humans) and spent more time lying down. Some monkeys showed a dramatically impaired performance on a bimanual motor skills (BMS) task, with slower times to perform the test or no interest at all.

Signs of PD-like disease were scored using the Kurlan scale [17], considered to be the optimal clinical rating scale for MPTP-induced Parkinsonism in macaques [18]. Through this dosing and rating system, we induced a state of mild stable Parkinsonism (Kurlan scores of 36.5 persisting a minimum of 7 weeks following the last dose, Table 1, Figure 1). Histopathological analysis of the dorsal striatum clearly showed a profound decrease in tyrosine hydroxylase (TyH) [Figure 2A (upper panel), 2B (upper panel)] as well as dopamine transporter (DAT) [Figure 2A (middle panel), 2B (lower panel)] staining in the MPTP-treated monkeys compared to untreated controls, revealing persistent damage to the pre-synaptic dopaminergic terminals from the $\mathrm{SN}$.

\section{Upregulation of Cat D mRNA and protein in MPTP-treated monkey $\mathrm{CN}$}

In order to assess whether neurodegenerative mechanisms were ongoing in the $\mathrm{CN}$ long after the termination of MPTP treatment, we assessed the expression of Cat D. We first analyzed if Cat D is altered at the mRNA level. We extracted RNA from CN of the MPTP treated monkeys and quantified Cat D mRNA by quantitative real-time PCR in comparison to caudate RNA from four untreated control monkeys. We found a significant increase $(\mathrm{p}<0.05)$ in Cat D mRNA levels in chronically MPTP-treated animals (Figure 3A).

Next, we investigated which cell types in $\mathrm{CN}$ show increased production of Cat $\mathrm{D}$ protein expression. As seen in Figure 3B, there is increased Cat D immunoreactivity in the neurons of MPTP-treated monkey $\mathrm{CN}$. Interestingly, there is increased staining in soma and axonal cones. Intriguingly, this staining pattern has been previously reported to be present in AD [15]. Furthermore, we examined co-localization of Cat D and the neuronal marker MAP2 and ascertained that 
Table 1 MPTP-treated animal subjects

\begin{tabular}{lllll}
\hline Animal & Total MPTP $(\mathbf{m g} / \mathbf{k g})$ & Weeks since first treatment & Weeks since last dose & Final Kurlan score \\
\hline 543 & 4.1 & 31 & 7 & 3 \\
547 & 4.1 & 31 & 7 & 3 \\
549 & 2.7 & 23 & 8 & 6.5 \\
\hline
\end{tabular}

Animals received intermittent low-dose MPTP as indicated in Figure 1. The total dose of MPTP received by each animal, total time since first treatment, the time between the last dose and animal sacrifice, and the clinical score at sacrifice is indicated.

its increased expression is indeed in neurons (Figure 4A). Additionally, we examined if Cat D co-localizes with IBA1, a microglial marker; however we did not observe distinct expression of Cat D in microglial cells (Figure 4B).
Proper regulation of cathepsin levels is important in neurons, and upregulation as well as absence of cathepsins have considerable consequences on the maintenance and function of nervous system. Lysosomal proteases are rarely secreted outside the cells under

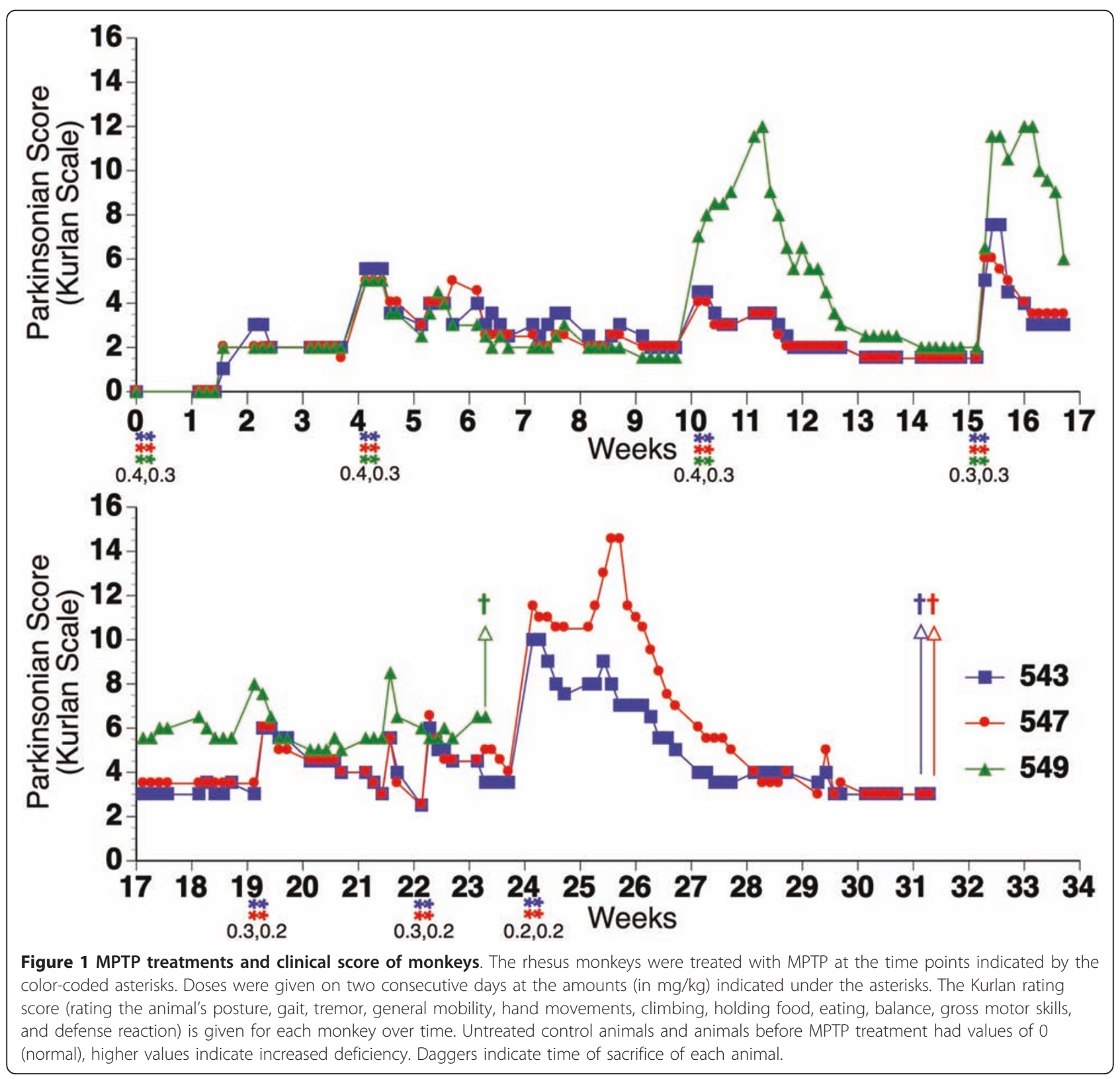




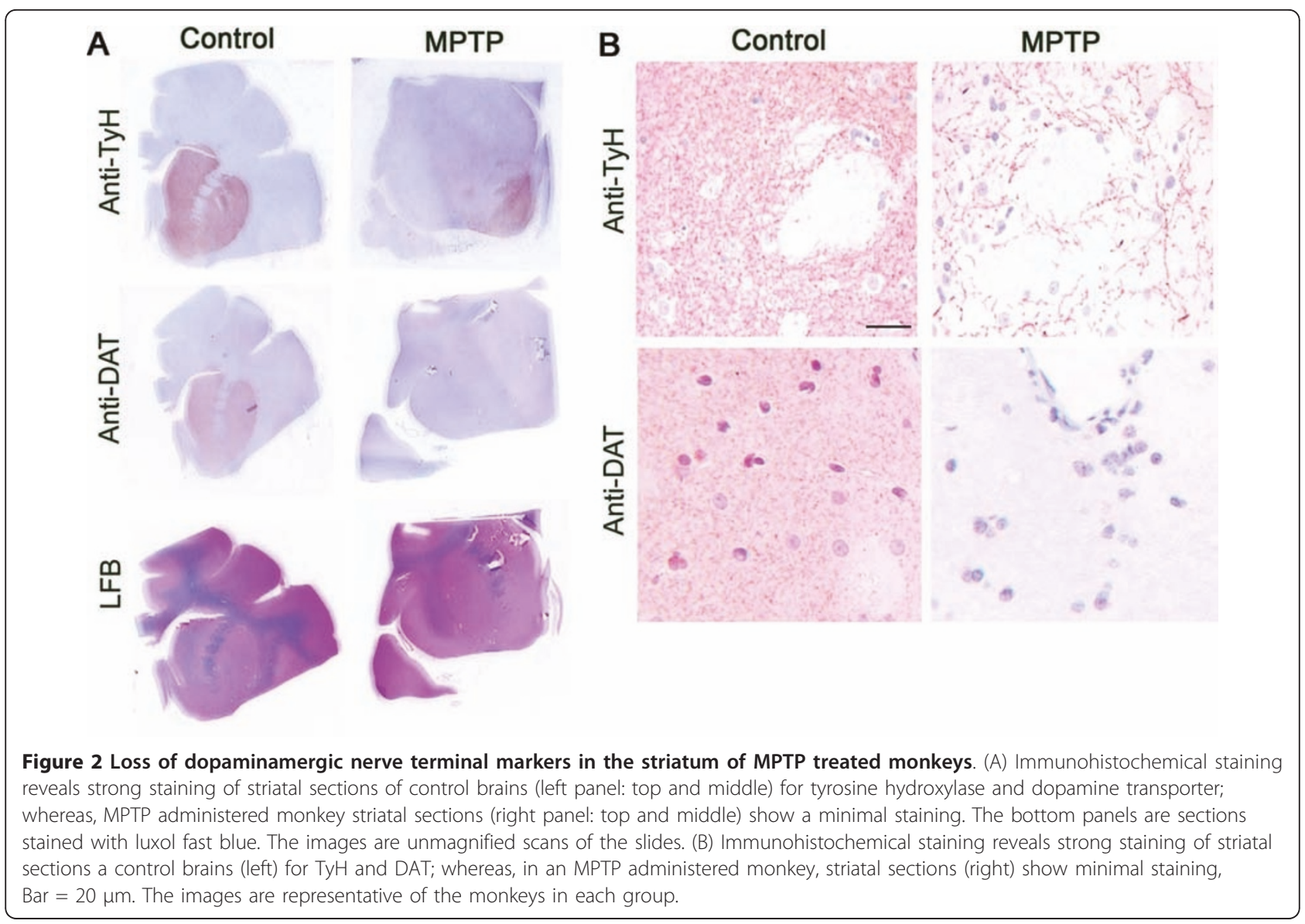

normal conditions. Normal levels of Cat D have the ability to digest $>95 \%$ of total brain protein within 24 hr under in vitro conditions [19], implicating that the leakage of lysosomal compartments can have profound deleterious effects on the cell itself and if released outside the cells would effect the surrounding brain milieu. To investigate the relationship of Cat D to lysosomes in the brains, we performed double immunofluorescence for Cat D and LAMP-2, a marker for lysosomes. As seen in Figure 5A (upper), normal neurons contain a few lysosomes in the neuronal cell body and Cat D is localized with the lysosomes. However in the MPTPtreated monkey $\mathrm{CN}$ sections (Figure 5A lower), we see a drastic increase in the number of lysosomes throughout the neuronal cell body as well as in Cat D immunoreactivity. Quantification reveals an average of four-fold increase in lysosomes (Figure 5B). Given this increase, we next questioned whether increased lysosomal biogenesis could arise from overexpression of Cat D.

\section{Changes in Cat D and lysosomes due to Cat D overexpression}

To investigate the effect of increased Cat D in neurons, we overexpressed human C-terminal GFP-tagged Cat D
(Cat D-GFP) or a control GFP (green fluorescent protein) construct in the BE-2 (M17) human neuroblastoma cell line. Using lysotracker red as an indicator of lysosomes in control BE-2 cells, we observed the presence of normal intracellular lysosomes (Figure 6A). In Cat D transfected cells, Cat D-GFP largely co-localizes with lysosomes, and the number of lysosomes was significantly increased relative to controls (Figure 6A, B), consistent with the findings in our in vivo model.

\section{Cat $D$ is present extralysosomaly and extracellularly}

We next asked if we could detect Cat D enzyme activity in the cytosol and extracellularly. We performed cytosolic fractionation from cellular organelles (Additional file 1) using BE-2 cells, either non-transfected, transfected with GFP alone, or transfected with Cat D-GFP. The Cat D expression as well as activity were measured in cytosolic extracts and in the culture supernatants of the Cat D transfected cells was indeed significantly greater when compared to controls. The active/mature form of Cat D was detected in cytosol (Figure 6C upper panel). Cat D activity assay showed an increase in Cat D activity in the cytosolic fractions as well (Figure 7A, B). Not only can the presence of cytosolic Cat D be deleterious 

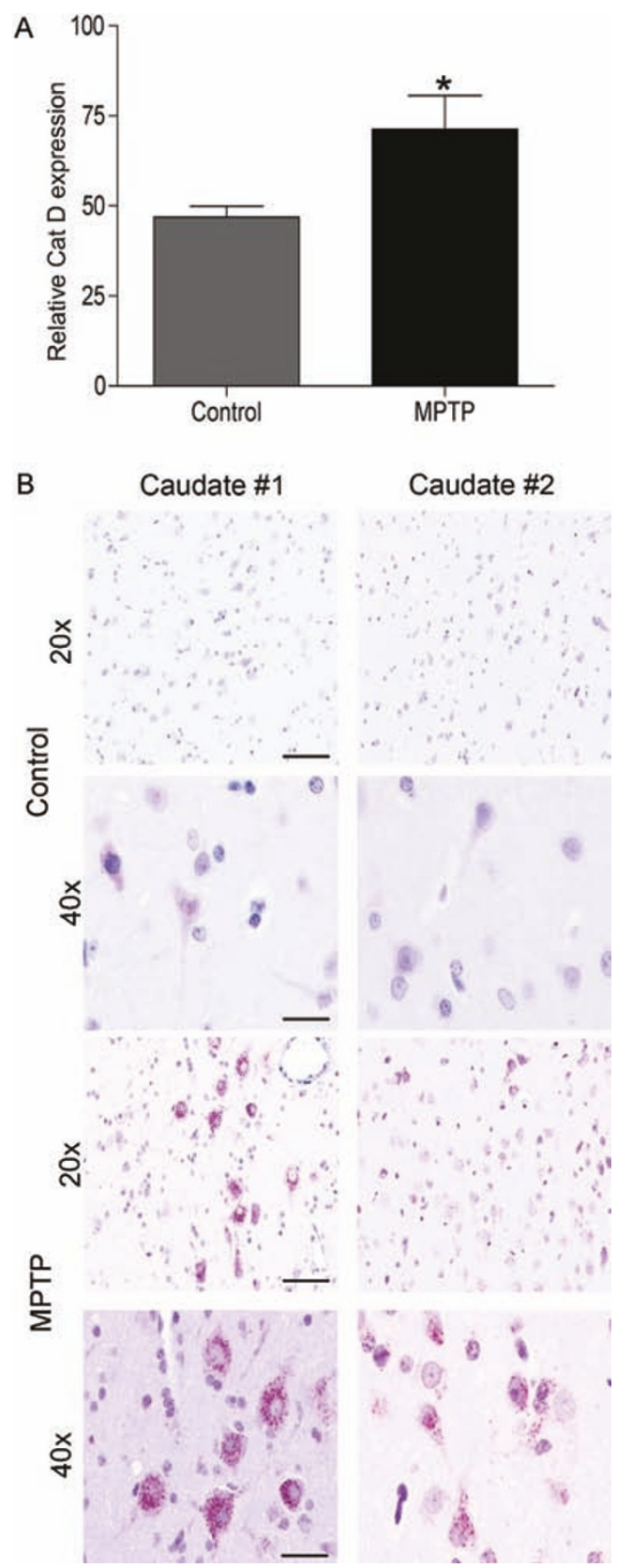

Figure 3 Cat D is upregulated in MPTP caudate nucleus. (A) Taqman qRTPCR for Cat D mRNA was performed on RNA from caudate samples. A significant upregulation in Cat D mRNA is seen. The delta $\mathrm{Ct}(\mathrm{dCt})$ method was performed to determine relative concentrations, using the average of the $\mathrm{Ct}$ of $18 \mathrm{~S}$ and GAPDH as the normalizing value. Mean and standard error of the mean shown, significance is indicated by ${ }^{*} p<0.05$ (Student's t-test). (B) Photomicrographs of representative sections of caudate of two animals (\#1, \#2) from each group of the control and MPTP monkeys Caudate sections were immunohistochemically stained with anti-Cat D. Minimal staining is seen in sections from control animals (bar $=$ $50 \mu \mathrm{m})$ and in corresponding increased magnification (40x, bar $=$ $10 \mu \mathrm{m})$; whereas, in MPTP treated animal, there is increased staining. Increased magnification $(40 \times$, bar $=10 \mu \mathrm{m})$ reveals that the staining is seen within neurons in the cell body, axon hillock (bottom right). to the cell itself but the presence of extracellular Cat D could be lethal to the surrounding cells. Thus, we subsequently questioned if Cat D expression triggered cell death.

\section{Overexpression of Cat D leads to caspase activation and apoptosis}

To investigate whether Cat D overexpression induces pathways leading to neuronal death, we first performed a lactate dehydrogenase (LDH) assay on the culture supernatant, assessing loss of plasma membrane integrity. Figure $8 \mathrm{~A}$ shows that there is a significant amount of LDH-release caused by overexpressing Cat D in rat striatal neurons when compared to controls. We next performed a fluorescence live imaging assay using the cell-permeable fluorescein isothiocyanate (FITC) conjugate of the caspase inhibitor VAD-FMK. This inhibitor irreversibly binds to the activated caspase, allowing for the in situ labeling of cells in which the caspase activation cascade has been initiated. As seen in Figure 8B, when compared to control, the Cat D transfected rat striatal neurons show a greater labeling for caspases, likely due to initiation of apoptosis. Interestingly, caspase activation is also seen along the neurites in Cat D transfected cells (see Figure $8 \mathrm{~B}$ ) indicating neuronal damage.

To evaluate the presence of apoptotic cells, Cat DGFP transfected and control BE-2 cells were stained with Hoechst stain (Figure 9A). The fragmentation of nuclei was clearly observed in Cat D transfected cells when compared to controls. To further strengthen our observation, we performed an in situ TUNEL assay. As seen in Figure 9B, fragmented DNA in Cat D transfected cells was clearly labeled. Also, since we found caspase activation, we looked for cytosolic release of Cytochrome C (Cyto-C), an activator for apoptosis. As seen in Figure 9C, there was a clear cytosolic mobilization of Cyto-C in Cat D overexpressing cells confirming its release, which is strongly linked to activation of the apoptotic pathway.

\section{Discussion}

In human PD a major unmet challenge has been to curb the progression of disease that can affect the higher cognitive functions of the patient. The molecular triggers that induce the progression of disease to areas like the $\mathrm{CN}$ and that contribute to associative and executive cognitive functions are currently unknown [2,20,21]. Using a nonhuman primate model of PD, we report for the first time that lysosomal instability and alterations in a lysosomal protease, Cat $\mathrm{D}$, in $\mathrm{CN}$ can lead to degeneration and dysfunction of neurons (Figure 10).

In the chronic MPTP model of PD, we first found a significant upregulation of Cat D in the CN. Importantly, 


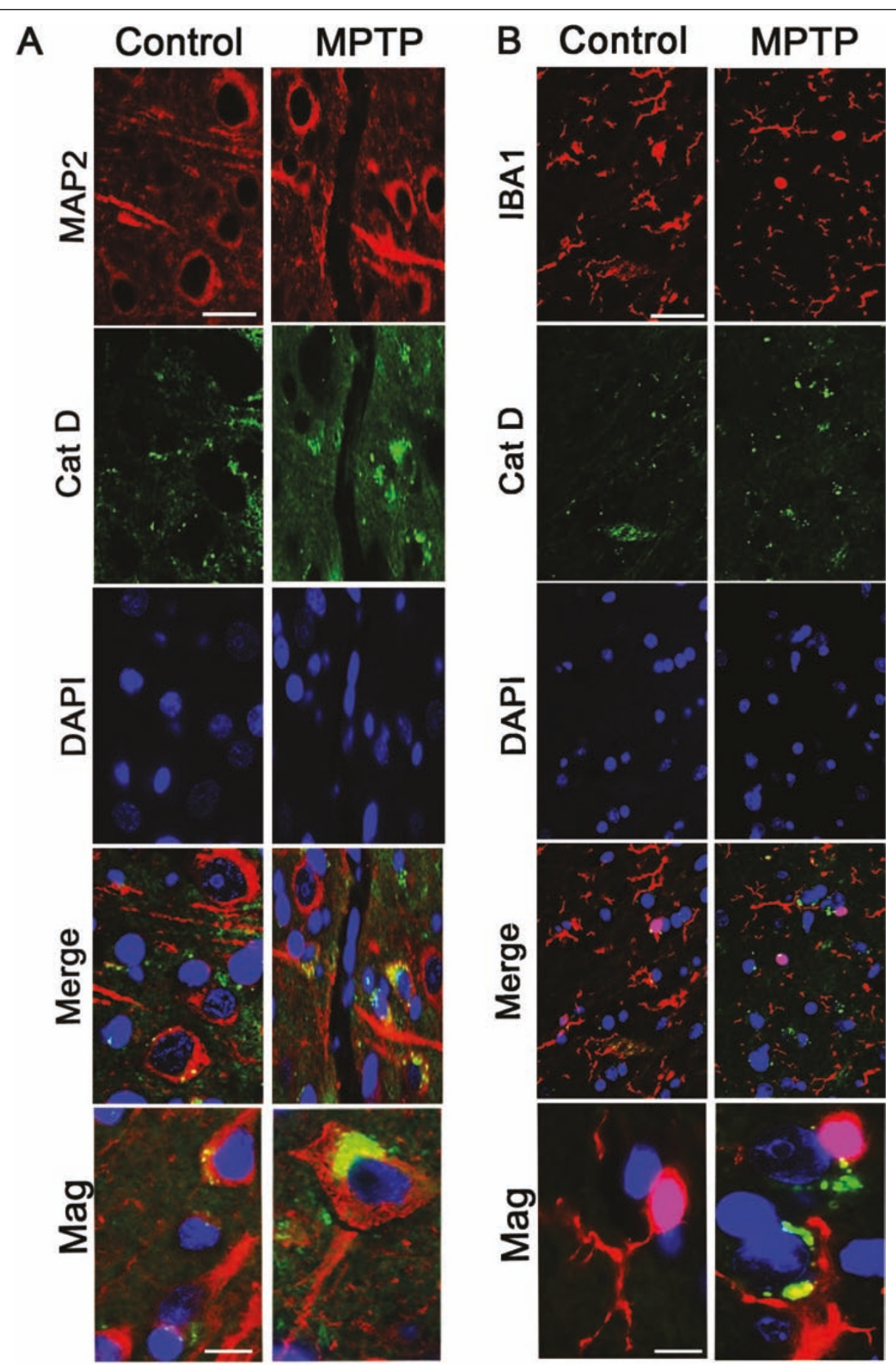

Figure 4 Double immunofluorescence of CatD with MAP2 and IBA1 in CN od MPTP monkey. (A) Double immunostaining was performed on MPTP CN sections with the neuronal marker anti-MAP2 (red) and anti-Cat D (green). The merged image illustrates a co-localization of Cat D with MAP2, confirming its presence in neurons. Bar $=20 \mu \mathrm{m}$. Higher magnification of single neuron is also provided (bottom panel, Mag). Bar $=$ $5 \mu \mathrm{m}$. (B) Double immunostaining was performed on MPTP CN sections with the microglial marker anti-IBA1 (red) and anti-Cat D (green). The merged staining of Cat D with IBA1 conforms the absence of Cat D in microglial cells. Bar $=20 \mu \mathrm{m}$. Higher magnification (bottom panel, Mag). Bar $=5 \mu \mathrm{m}$. 


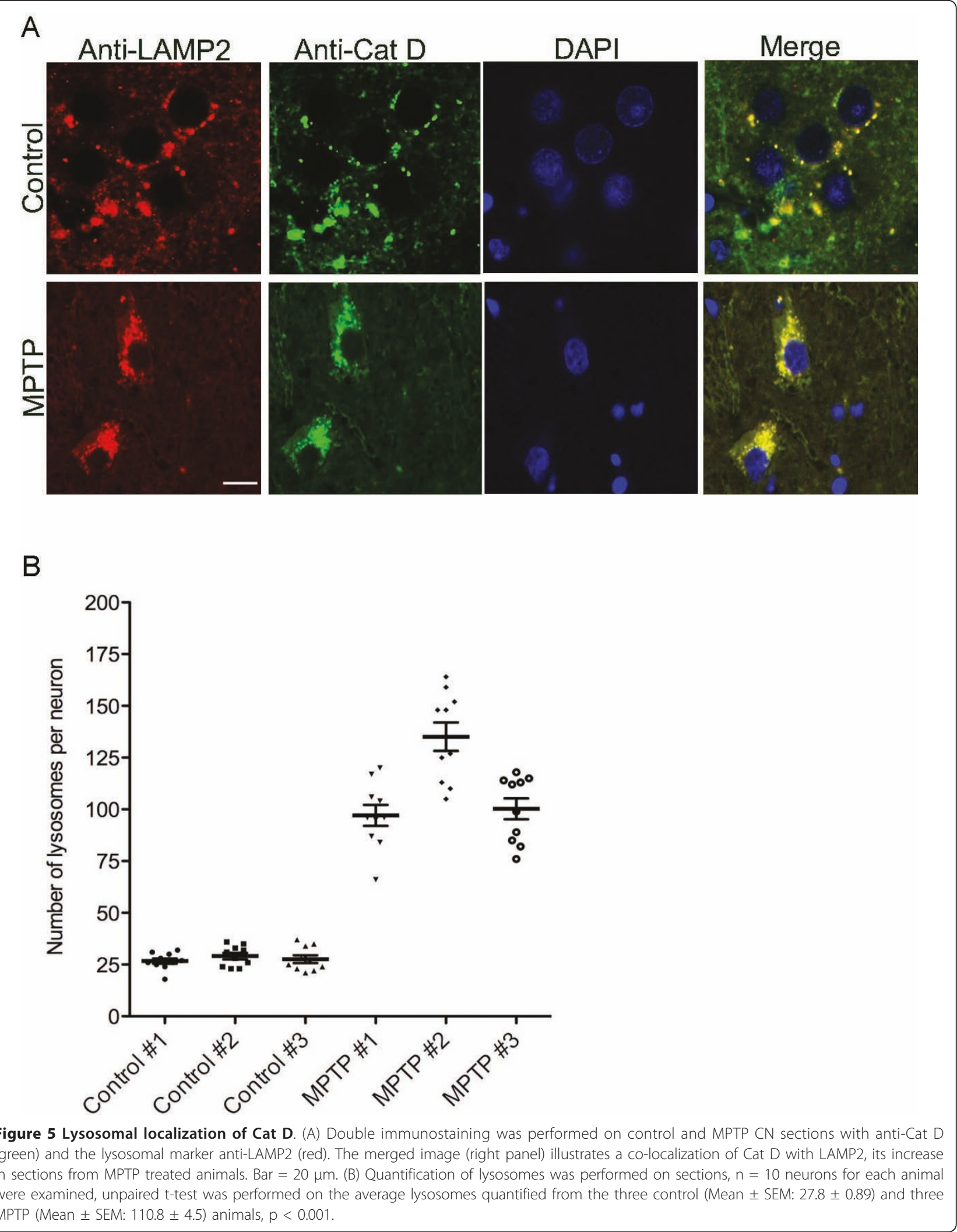




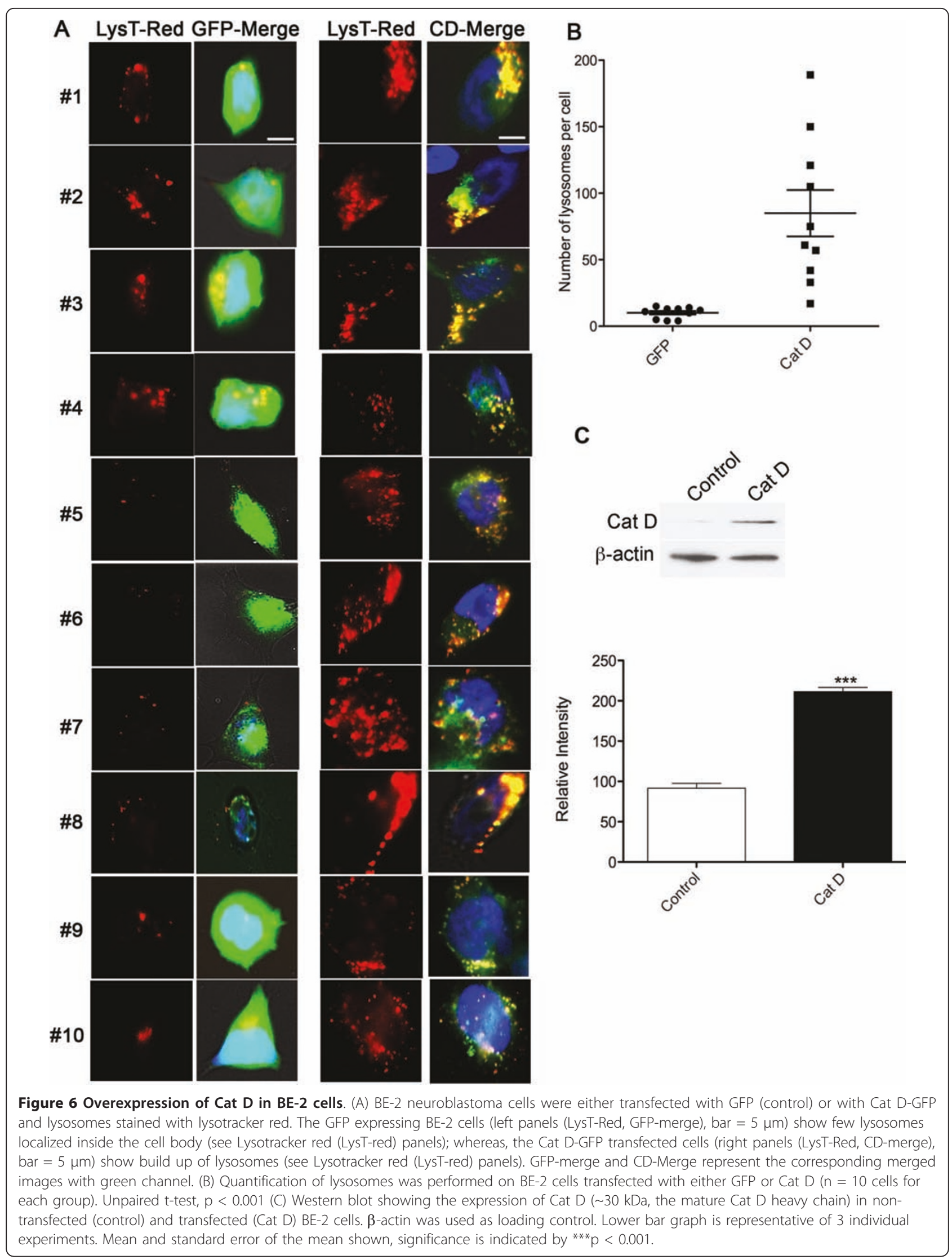




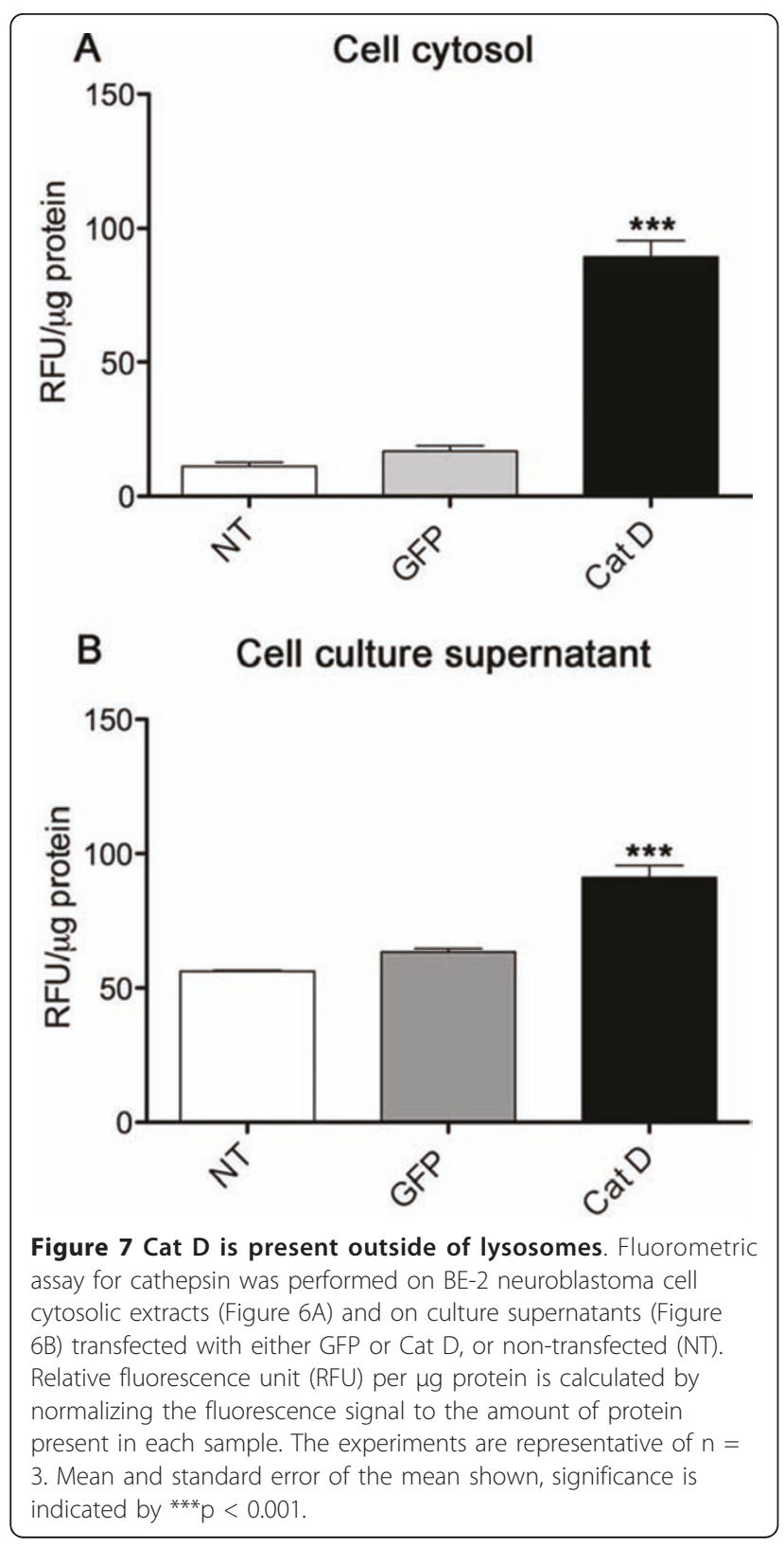

we also observed that there is an increased staining for Cat $\mathrm{D}$ in neurons, and an increase in the number of lysosomes in neurons in MPTP-induced PD. In vitro we found that Cat D overproduction itself led not only to increased lysosomal biogenesis but also led to the occurrence of extralysosomal (including extracellular) Cat D and caspase activation leading to the activation of apoptotic pathway.

While the trigger for increased Cat D production is not known, it has been shown that in order to compensate for the loss of DA in PD, an increased rate of DA

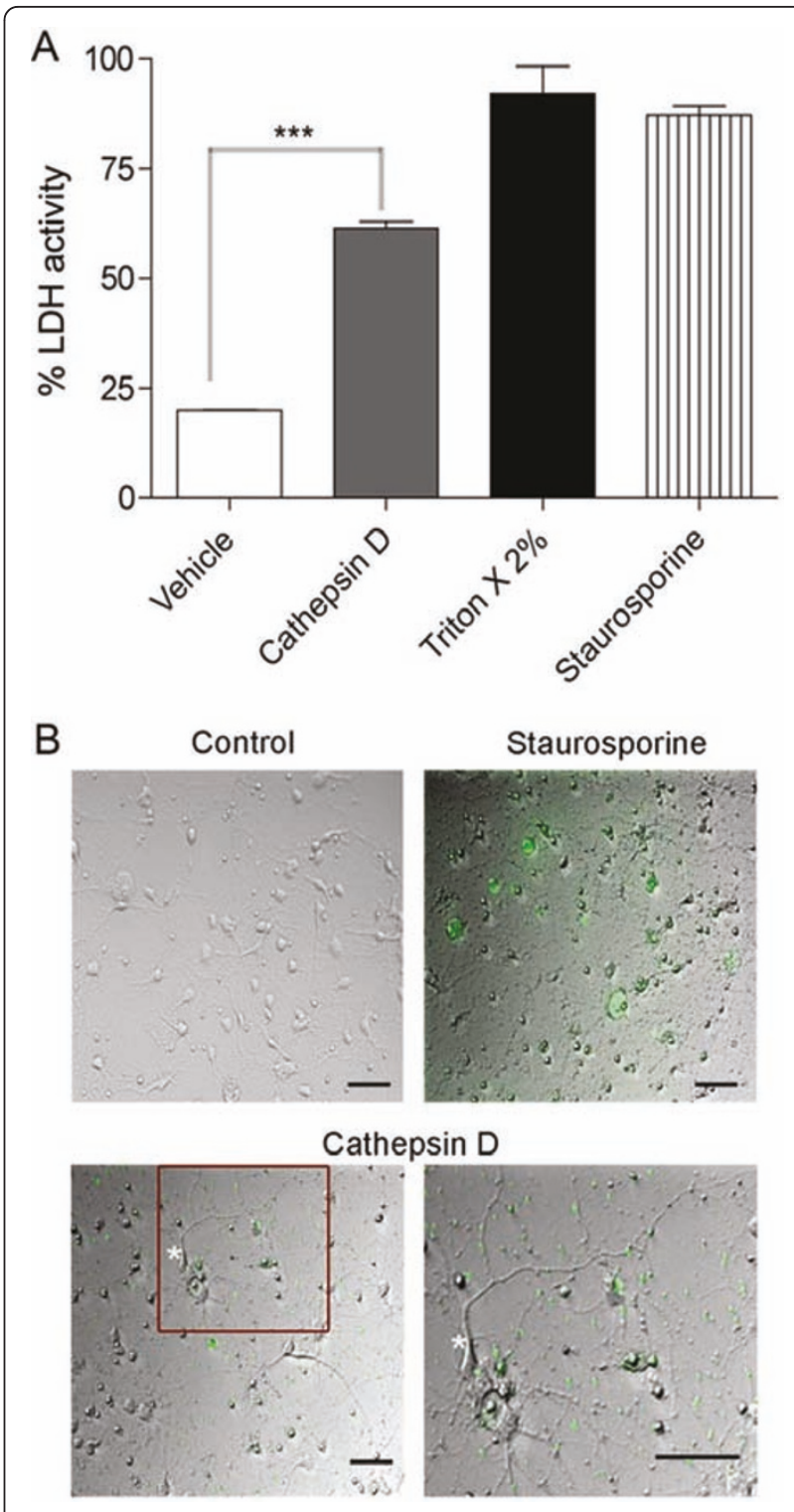

Figure 8 Cat $D$ overexpression leads to release of lactate dehydrogenase (LDH) and caspase activation. (A) LDH assay was performed on the rat striatal neurons transfected with either vehicle alone or Cat D. For positive control, 2\% Triton X-100 and staurosporine treatments were performed. Cat $\mathrm{D}$ overexpression significantly increased the amount of $\mathrm{LDH}$ in media. The experiment is representative of $n=4$. Mean and standard error of the mean shown, significance is indicated by ${ }^{* *} p<0.001$. (B) Live imaging performed on rat striatal neurons. Neurons were transfected with Cat D and stained for CaspACE FITC-VAD-FMK in situ marker. The green staining represents the activation of caspase and apoptosis. As seen, compared to control the Cat D transfected neurons show a clear activation in FITC caspase levels, including in neurites (boxed area in lower left panel enlarged in lower right, see processes on neuron marked by asterisk). Staurosporine treated neurons were used as a positive control. Bar $=50 \mu \mathrm{m}$. 


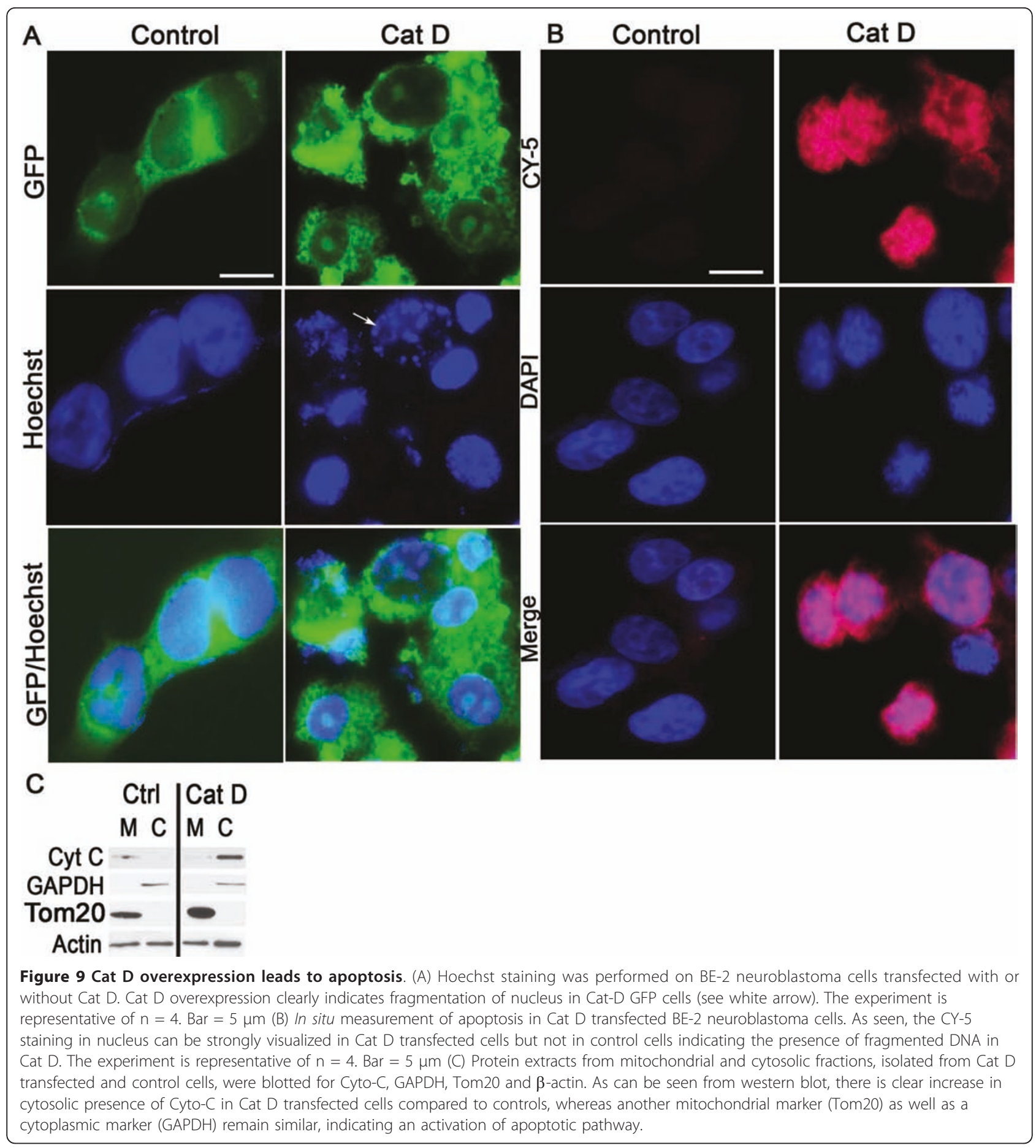

turnover takes place in the striatum, especially in the $\mathrm{CN}$. Therefore, these hyperactive neurons are prone to more oxidative stress [4]. Intriguingly, oxidative stress is thought to activate lysosomes and release Cat D into the cytosol in non-neuronal cells [22-26]. Although oxidative stress has been described as a main trigger for the loss of DA neurons in $\mathrm{SN}$ pars compacta region in $\mathrm{PD}$ [27], its link to PD-related changes in other parts of the brain are less well studied. A complete breakdown of lysosomes and release of high concentrations of lysosomal enzymes can lead to necrosis; whereas partial or selective release of lysosomal proteases could trigger apoptosis [28]. Studies in non-neuronal cells have shown that lysosome membrane permeabilization followed by Cat D release are actually important events in the death cascade and occurs downstream of the Bax- 


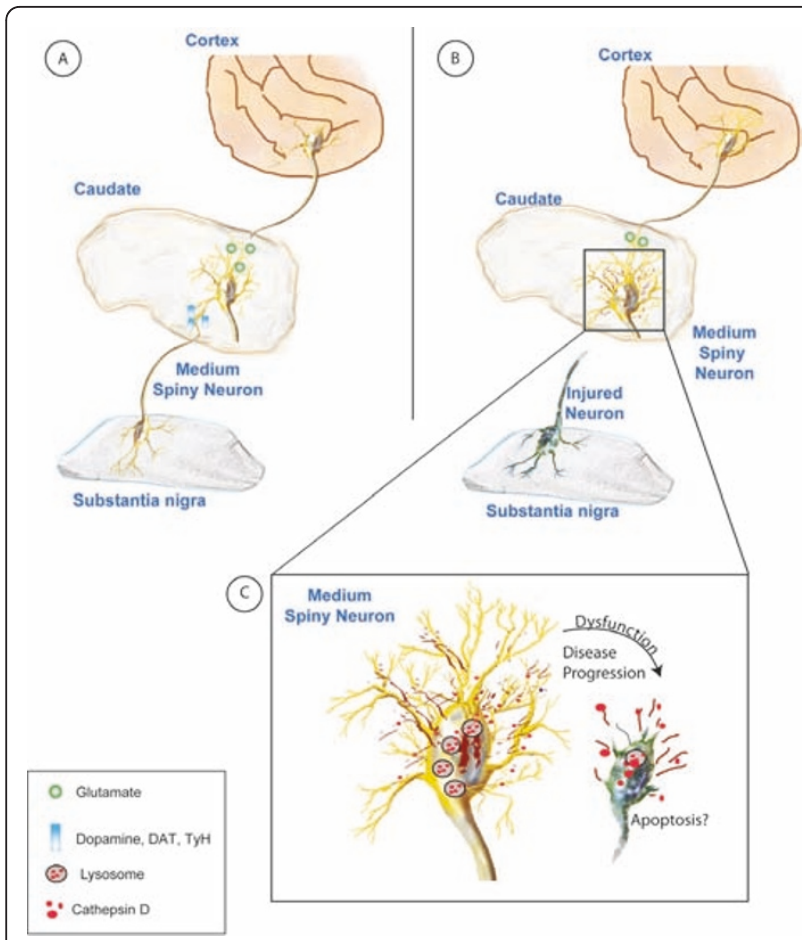

Figure 10 Schematic representation of disease progression in the caudate. (A) Depiction of the neuronal pathways involved in normal brain functioning, glutamatergic as well as dopaminergic innervations from cortex and substantia nigra (SN) to the caudate nucleus. (B) MPTP and PD affects the neurons in SN leading to loss of the dopaminergic projections to the caudate, leading to dysregulation of lysosomal pathways, and increasing the number of lysosomes as well as Cat D and extralysosomal Cat D. (C) Slow but persistent damage resulting from upregulation of Cat $\mathrm{D}$ expression and leakage leads to neuronal dysfunction and death through apoptosis or other mechanisms.

dependent caspase mechanism [29] linking the apoptotic machinery to neuronal loss in our study.

Cell death has been extensively examined in PD, but most of these studies relate to direct DA neuronal loss in the $\mathrm{SN}$ pars compacta region, the initiating site of disease [30]. However, we examined the $\mathrm{CN}$, which is not the direct site of injury caused by MPTP but is affected by the progression of disease. Here, we identify the activation of lysosomal pathways as pathogenic in the $\mathrm{CN}$.

Intriguingly, not only was Cat D upregulated in neurons but also was seen to be present in the cytosol and extracellularly, a phenomenon that has not been examined until now in PD or its models. By overexpressing Cat D in human neuroblastoma cells or in rat striatal neurons, we see a similar phenomenon. The deleterious effects of protease leakage in particular, Cat D, into the extracellular environment has been best addressed in breast cancer research where studies indicated that the extracellular matrix was degraded by Cat D released from cancer cells into an acidic microenvironment consequently facilitating tumor invasion [31]. Cancer related studies have also revealed that the presence of cytosolic CD stimulates apoptotic pathways by interacting with members of the apoptotic machinery [32]. Recently, studies also suggest that pro-cat-D secreted by epithelial cancer cells promotes fibroblast outgrowth in a paracrine LRP1-dependent manner in the breast tumor microenvironment [33].

There is precedence for demonstration of extralysosomal Cat D in neurodegeneration. Trimethyltin chloride (TMT) is a potent neurotoxic agent. TMT was shown to upregulate Cat D in neurons both in vivo and in vitro, and following TMT treatment of rats Cat D is present in the neuronal cytoplasm, and linked to apoptosis [34]. Gaucher's disease is the most common form of lysosomal storage disorders, results in profound neuronal damage, and can be modeled in glucosylceramidase knockout mice. In neurons of such mice Cat D is upregulated and present in the cytosol, and its presence linked to neuronal damage [35]. Alzheimer's disease is the most prevalent neurodegenerative disorder, and aspects can be mimicked in transgenic mice through the expression of mutant forms of amyloid precursor protein in neurons, resulting in intraneuronal amyloid beta accumulation. This again is accompanied by Cat D leakage from lysosomes into the cytoplasm [36].

While our study as well as these support the mechanism that Cat D leakage leads to activation of apoptotic pathway and then cell death, we cannot exclude that Cat D leakage itself can lead to other causes of cellular destruction such as through proteolysis. Lysosomal membrane permeabilization, which includes leakage of Cat D, can result in an apoptotic death as well as cell death that has a subapoptotic or necrotic characteristic [37]. Furthermore there is the possibility that the Cat D leakage is a result of neuronal damage, and not its cause. Although our in vitro experiment does not directly address the cause of the leakage phenomenon in vivo, the increased Cat D mRNA as well as protein expression definitely is indicative of dysregulated lysosomal pathways, and this could be addressed more effectively in additional animal studies in the future. While cognizant of these caveats, our data provides evidence that leakage of Cat D can be the cue for spread of disease to other regions of the brain in PD.

While others have suggested that increasing expression of Cat D could be used as a therapeutic strategy in PD as it clears toxic $\alpha$-synuclein aggregates [38], our data clearly indicate that over-production or increased Cat D levels in neurons can lead to neuronal injury, raising caution to this approach, as well as providing pathogenic clues to the progressive neuronal damage in PD. 


\section{Conclusions}

In conclusion, our study provides strong evidence that Cat $\mathrm{D}$ can be involved in the long-term neuronal damage in PD in regions remote from the site of primary insult, and underscores the need to study brain regions outside the sites of initial damage in neurodegeneration to help understand the basis of disease progression. Therefore, this study provides new avenues for therapeutic interventions that might help to treat or arrest the progression of motor and cognitive disorders commonly seen in human PD patients.

\section{Materials and methods \\ Animals}

Adult (6-8 years of age) male rhesus monkeys served as subjects or controls for this study. Monkeys, free from SIV (simian immunodeficiency virus), type D simian retrovirus, and herpes $\mathrm{B}$ virus were obtained from Covance (Alice, TX, USA) and Valley Biosystems, Inc. (Sacramento, CA, USA). Animals were kept in a biocontainment facility and housed individually in stainless-steel cages, which permitted olfactory, visual, and auditory contact with other monkeys in the room. Monkeys were paired housed during several hours of the day, which permitted animal interaction and evaluation of social behavior. The room was maintained on a 12/12 hr light/ dark cycle (lights on 0600; lights off 1800) and at a temperature of $25 \pm 1^{\circ} \mathrm{C}$. Monkeys had previously been trained on a BMS task as previously described [39]. The task was designed to test bimanual motor coordination, procedural learning and motivation to work for a preferred food (raisins). Due to the hazardous nature of MPTP, the treated animals (\#543, \#547 and \#549) were kept together in a separate room in the facility. The control animals (\#516, \#517 and \#546) did not receive vehicle injections; they did receive ketamine injections for blood draws. The controls were chosen to be of equivalent age as the experimental group.

\section{MPTP treatment of nonhuman primates}

MPTP hydrochloride (Sigma-Aldrich, St. Louis, MO, USA) was dissolved in sterile saline and given intramuscularly to lightly anesthetized ( $5 \mathrm{mg} / \mathrm{kg}$ ketamine) rhesus monkeys on two consecutive days at an initial dose of $0.4 \mathrm{mg} / \mathrm{kg}$. For clinical rating of Parkinsonism, the Kurlan scale was used in which monkeys were rated daily on weekdays for posture, gait, tremor, general mobility, hand movements, climbing, holding food, eating, balance, gross motor skills, and defense reaction as described [17] in addition to BMS. Animals were tested until recovery from acute effects of MPTP were determined by a stable rating score and were evaluated for the next round of dosing, which was given at doses of $0.3-0.4 \mathrm{mg} / \mathrm{kg}$. The time scale of dosing is indicated in
Figure 1, and the overall amount given to each animal is listed in Table 1.

All experiments were performed under the approval of the Environmental Health and Safety Department, the Institutional Biosafety Committee and the Institutional Animal Care and Use Committee of The Scripps Research Institute, following NIH guidelines.

\section{RNA isolation and Quantitative Real Time PCR}

The $\mathrm{CN}$ was dissected from the brains, snap frozen, and RNA isolated using Trizol (Invitrogen, Carlsbad, CA, USA). RNA was quantified and determined to be of good purity by spectrophotometric analysis on a NanoDrop 8000 (Thermo Scientific, Wilmington, DE, USA). To check the integrity, RNA samples were run on the Bioanalyzer 2100 (Agilent Technologies, Santa Clara, CA, USA). The RIN values in the range of 7-9 for the RNA samples were indicative of good quality.

Two micrograms of RNA from the $\mathrm{CN}$ of control and MPTP monkeys were used for reverse transcription. In a $50 \mu \mathrm{L}$ reaction, reverse transcription (RT) was carried out using the Superscript kit (Invitrogen) for $1 \mathrm{hr}$ at $42^{\circ}$ $\mathrm{C}$, followed by $70^{\circ} \mathrm{C}$ for $5 \mathrm{~min}$ to inactivate the RT reagents. RNase $\mathrm{H}$ (New England Biolabs, Beverly, MA, USA) treatment was then performed at $37^{\circ} \mathrm{C}$ for $20 \mathrm{~min}$. An equal volume of RNase and DNase free water was then added to the reactions.

Real time PCR was performed using gene-specific primers and probes. The primers and probe sequences were designed for rhesus sequences using the Genescript online tool http://www.genescript.com and obtained from Eurogentec (San Diego, CA, USA). The sequences of the primers and probe for $18 \mathrm{~S}$ and GAPDH controls have been previous reported [40]; whereas, the ones for Cat D were based on the rhesus Cat D sequence (GenBank accession XM_001091601.2): forward primer ACTGCAAACTGCTGGACATC, reverse primer CGTAGTGGATGGCAAATGAG, probe CAGCGACAAGTCCAGCACCTACG. Dilution analysis was carried out using monkey spleen cDNA on all the primers used for the study. The resulting $\mathrm{Ct}$ values indicated good linearity over the dilutions, with $\mathrm{R}^{2}$ values ranging from 0.994 to 0.998 , and the efficiencies calculated from the slopes were from 95 to $100 \%$.

To carry out quantitative real time PCR, $2 \mu \mathrm{L}$ of the (1:10 diluted) cDNA was used for assaying the amount of $18 \mathrm{~S}$ endogenous rRNA; $5 \mu \mathrm{L}$ (undiluted) each for all other genes. All reactions were performed in duplicates, $12.5 \mu \mathrm{L}$ of Taqman gene expression master mix (Applied Biosystems, Foster City, CA, USA) was added per $25 \mu \mathrm{L}$ reaction. The reaction mixture was brought to a final concentration of $5 \mathrm{mM} \mathrm{MgCl}$. Real time PCR was performed in 96-well plate on a StepOnePlus real time PCR system (Applied Biosystems). The delta Ct 
$(\mathrm{dCt})$ method was performed to determine relative concentrations using the average of the $\mathrm{Ct}$ of $18 \mathrm{~S}$ and GAPDH as the normalizing value. Relative units $\left(2^{\mathrm{dCt}}\right)$ were calculated and used as a measure of mRNA expression. Unpaired student's $t$ tests (GraphPad Prism Software, San Diego, CA, USA) were used for statistical analyses.

\section{Immunohistochemistry and Double Immunofluorescence}

Formalin-fixed, paraffin-embedded tissue blocks containing the striatum (caudate nucleus and putamen) were sectioned at $5 \mu \mathrm{m}$ thickness and picked up on glass slides. For immunohistochemistry, following deparaffinization, antigen retrieval was performed by heating to $95^{\circ} \mathrm{C}$ in $0.01 \mathrm{M}$ citrate buffer, $\mathrm{pH} 6.39$, for $40 \mathrm{~min}$, then left for $20 \mathrm{~min}$ to steep. Sections were blocked in $0.5 \%$ casein, followed by the addition of either 1:1000 dilution of an antibody to $\mathrm{TyH}$ (Millipore, Billerica, MA, USA), 1:1000 dilution of an antibody to DAT (Millipore) or 1:50 dilution of an antibody to Cat D (Cell Signaling, Denvers, MA, USA) overnight at $4^{\circ} \mathrm{C}$. Following washes, signal was detected using the SuperPicture broad spectrum secondary antibody-horseradish peroxidase polymer reagent (Invitrogen) and developed with the 3,3'-Diaminobenzidine (DAB) (Vector Laboratories, Burlingame, CA, USA), followed by a hematoxylin counterstain (Invitrogen) and examined microscopically (Zeiss inverted microscope, Carl Zeiss, NY, USA).

For double immunofluorescence, sections were deparaffinized, antigen retrieval was performed by heating to $95^{\circ} \mathrm{C}$ in $0.01 \mathrm{M}$ citrate buffer, $\mathrm{pH} \mathrm{6.39}$, for $40 \mathrm{~min}$, then left for $20 \mathrm{~min}$ to steep. Sections were blocked in $20 \%$ BSA, followed by the addition of a 1:50 dilution of rabbit polyclonal anti-Cat D antibody (Cell Signaling) overnight at $4^{\circ} \mathrm{C}$. Following PBS washes, sections were incubated in chicken anti-rabbit Alexa-Flour 488 secondary antibody (Invitrogen) for $1 \mathrm{hr}$, followed by blocking in 20\% BSA and incubation with 1:100 dilution of rat monoclonal anti-LAMP2 antibody (Abcam, Cambridge, MA, USA) overnight at $4^{\circ} \mathrm{C}$. Following washes, sections were incubated in goat anti-rat Alexa-Flour 568 secondary antibody (Invitrogen) for $1 \mathrm{hr}$ followed by final washes, mounting in Prolong gold anti-fade with DAPI (Invitrogen). For MAP2 double staining, following staining and detection of Cat D, mouse monoclonal anti-MAP2 (Sternberger Monoclonals, Covance, Princeton, NJ, USA) antibody was used in conjunction with goat-anti-mouse Alexa-Flour 568 secondary antibody (Invitrogen). For microglial double immunofluorescence, the above staining protocol was followed, except using mouse monoclonal anti-Cat D (Abcam) followed by goat-anti-mouse Alexa-Fluor 568 as a secondary antibody, and rabbit polyclonal anti-IBA1 (Wako, Richmond, VA, USA) followed by goat-anti-rabbit Alexa-Fluor 488 secondary antibody (Invitrogen). We interchanged the IBA1 color to red and Cat D to green using the AxioVision REL 4.8 Software (Carl Zeiss, NY, USA) so that the images appear consistent throughout the panel.

\section{Quantification of lysosomes}

Images were taken as $z$-stacks at $63 \times$ for larger magnification. A single neuron was selected from the image, and a final merged image was generated (Cat D/ LAMP2). The number of round lysosomes in each plane of the $z$-stack was quantified using a manual counting system recommended by the software. Each plane was scanned for presence of lysosomes, which might be overlayed or hidden in different planes. To prevent duplication, the software generates an identification number for each lysosome. To allow the end-user to accurately determine the presence of a single large vesicle or multiple small ones, double-labeled lysosomes were quantified geometrically as well as densitometrically. Quantification was performed using imaging program, AxioVision REL 4.8 Software (Carl Zeiss, NY, USA). The data were analyzed in Graphpad Prism (GraphPad Software, La Jolla, CA, USA).

\section{Cell culture}

Human neuroblastoma BE-2 (M17) cells (American Type Culture Collection, Manassas, VA, USA) were cultured under sterile conditions in Dulbecco-modified Eagle medium (LONZA BioWhittaker, Portsmouth, NH, USA) supplemented with $10 \%$ fetal calf serum, penicillin (100 units $/ \mathrm{ml}$ ), and streptomycin $(100 \mu \mathrm{g} / \mathrm{ml})$ in $5 \%$ $\mathrm{CO}_{2}$ humidified atmosphere at $37^{\circ} \mathrm{C}$.

For isolation of primary rat neurons, embryonic (gestational day 18) rat striatum was purchased from Brain Bits LLC (Springfield, IL, USA). Neuronal cultures were prepared by dissociating the tissue with $0.25 \%$ trypsin for $30 \mathrm{~min}$, neutralized with $10 \%$ fetal bovine serum, and further dissociating by triturating. The resulting single-cell suspension was centrifuged (1000 $\mathrm{rpm} / 5 \mathrm{~min}$ ) and was cultured on poly-D-lysine coated plates in Neurobasal media containing $0.5 \mathrm{mM}$ l-glutamine, $50 \mu \mathrm{g} / \mathrm{ml}$ penicillin and streptomycin and supplemented with B27 (Invitrogen). The striatal neurons were grown for 11 days in vitro (DIV) and were stained with anti-TyH (Millipore) and anti-DAT (Millipore) to confirm purity.

\section{Transfections and imaging of rat striatal neurons and BE- 2 (M17) cells}

After DIV 11 in culture, the rat striatal neurons were transfected with $1 \mu \mathrm{g}$ human Cat D tagged with GFP at the carboxy-terminus (Origene Technologies, Rockville, MD, USA) or a GFP-only vector (Origene) by NeuroMag 
according to manufacturer's protocol (OZ Biosciences, Marseille, France). Neurons were cultured for $48 \mathrm{hr}$ after transfection and checked microscopically for GFP expression.

Transfection of BE-2 (M17) cells were done using Fugene 6 (Roche Applied Science, Indianapolis, IN, USA) as per manufacturer's instructions. After transfections, cells were fixed with 4\% PFA for $15 \mathrm{~min}$. Double immunoflourescence was performed on the lysotracker treated cells; cells were washed 3 times with $1 \times$ PBS for 5 min each wash. Cells were blocked in 10\% normal goat serum, followed by the addition of a 1:50 dilution of rabbit polyclonal anti-Cat D antibody (Cell Signaling) overnight at $4^{\circ} \mathrm{C}$. Following washes, cells were incubated in goat anti-rabbit Alexa-Flour 488 secondary antibody (Invitrogen) for $1 \mathrm{hr}$ followed by final PBS washes, mounting in Prolong gold anti-fade with DAPI (Invitrogen). Double-labeled lysosomes were quantified as described in this methods section.

\section{Cathepsin D (Cat D) enzyme activity}

Cat D activity was measured in cytosol and in cell culture supernatants of BE-2 (M17) cells transfected with Cat D. Cytosol was prepared by lysing cells in a hypotonic buffer (0.32 M sucrose, $10 \mathrm{mM}$ Pipes $\mathrm{pH}$ 7.4, 0.1 $\mathrm{M} \mathrm{NaCl}, 3 \mathrm{mM} \mathrm{MgCl}$, $5 \mathrm{mM}$ EDTA and $0.5 \%$ Triton $\mathrm{X}-100)$. Cell debris and nuclei were spun down at a low speed, $800 \times \mathrm{g}$ for $10 \mathrm{~min}$, and the supernatants were spun at a high speed, $100,000 \times \mathrm{g}$ in a TLA100.4 for 1 $\mathrm{hr}$. The supernatants contain the cytosolic contents, and the pellet contains other sub-cellular organelles.

Cat $\mathrm{D}$ activity was measured by using a kit containing a fluorogenic peptide substrate peptide, GKPILFFRLK (Dnp)-DR-NH2 labeled with MCA (Abcam, Cambridge, MA, USA). Reactions were initiated by the addition of substrate, and kinetics of substrate hydrolysis was measured using a fluorescent plate reader (Ex $340 \mathrm{~nm}$, Em $460 \mathrm{~nm}$ ). Data were imported to Graph Pad Prism for analysis and normalization to total protein assayed.

\section{Lactate dehydrogenase (LDH) assay and CaspACE FITC live-dead assay}

LDH-assay was performed as per kit instructions (Cytotoxicity Detection Kit, Roche). Briefly, rat striatal neurons were transfected with Cat $\mathrm{D}$; a vehicle treated was used as a control. As a high control, some neurons were exchanged with assay medium containing $2 \%$ Triton X100 , and only assay medium was measured as low control or background control. Some neurons were also treated with $0.5 \mathrm{mM}$ staurosporine (Roche), a positive modulator of cell death. An equal amount of reaction mixture was added to assay medium, and LDH release was measured spectroscopically at $490 \mathrm{~nm}$ using a fluorescent plate reader.
For live imaging of apoptotic neurons, CaspACE FITC-VAD-FMK in situ marker (Promega, Madison, WI, USA) was used. Briefly, the transfected neurons were incubated with $10 \mathrm{mM}$ of CaspACE FITC-VADFMK at $37^{\circ} \mathrm{C}$ for $30 \mathrm{~min}$. Cells were rinsed in PBS and examined in a fluorescence microscope (Zeiss inverted microscope).

\section{TUNEL staining}

TUNEL staining was performed using the ApopTag Plus Peroxidase In situ Apoptosis Detection kit (Millipore) according to the manufacturer's instructions with minor modifications. Briefly, cells were fixed on coverslips with $1 \%$ paraformaldehyde, followed by washes and post-fixation for $5 \mathrm{~min}$ at $-20^{\circ} \mathrm{C}$ with ethanol:acetic acid $(2: 1)$, followed by PBS washes. Samples were oxidized for 5 min with $3 \% \mathrm{H}_{2} \mathrm{O}_{2}$ in PBS to reduce endogenous peroxidase activity and then washed with PBS. Slides were next prehybridized in equilibration buffer for $5 \mathrm{~min}$, followed by hybridization with terminal deoxynucleotidyl transferase enzyme for $1 \mathrm{hr}$ at $37^{\circ} \mathrm{C}$. The hybridization reaction was terminated by incubation with stop buffer and PBS wash. HRP-conjugated anti-digoxigenin was added next (30 min at RT), followed by PBS wash. The signal was converted to fluorescence using TSA Cy5 kit (Perkin Elmer, MA, USA) according to the manufacturer's protocol. Coverslips were mounted in Prolong gold anti-fade reagent with DAPI (Invitrogen).

\section{Western blotting}

SDS-PAGE electrophoresis was performed using NuPAGE gel system (Invitrogen, Carlsbad, CA) in 4$12 \%$ gradient gels under reducing conditions. For western blot analyses, $10 \mu \mathrm{g}$ of protein extracts were loaded per lane. Nonspecific antibody binding was blocked using 5\% nonfat dried milk for $1 \mathrm{hr}$ at room temperature. Immunoblotting was carried out with polyclonal rabbit anti-Cat D (1:1000, Cell Signaling), monoclonal rabbit anti-LAMP1 (1:1000, Cell Signaling), or polyclonal rabbit anti- $\beta$-actin (1:5000, Thermo Fisher Scientific); followed by secondary antibody (1:20,000 HRPconjugated anti-rabbit IgG; Thermo Fisher Scientific). Blots were developed with 1:1 solution of Super Signal West Pico Chemiluminescent Substrate and Luminol/ Enhancer (Thermo Fisher Scientific, Rockford, IL, USA).

\section{Additional material}

Additional file 1: Figure S1.

\section{Abbreviations}

AD: Alzheimer's Disease; BMS: bimanual motor skills; Cat D: cathepsin D; CN: caudate nucleus; CytC: cytochrome C; DA: dopamine; DAB: 3,3'- 
Diaminobenzidine; DAB: 3,3'-Diaminobenzidine; DAT: dopamine transporter; $\mathrm{dCT}$ : delta CT; DIV: days in vitro; FITC: fluorescein isothiocyanate; GFP: green fluorescence protein; L-DOPA: L-3,4-dihydroxypheylalanine; LDH: lactate dehydrogenase; MPTP: 1-methyl-4phenyl-1,2,3,6-tetrahydropyridine; NT: nontransfected; PBS: phosphate buffered saline; PD: Parkinson's disease; RFU: relative fluorescence unit; RT: reverse transcription; SN: substantia nigra; TMT: trimethyltin chloride; TyH: tyrosine hydroxylase

\section{Acknowledgements}

This work was supported by NIH grants P30 MH062261 and R01 MH073490 in addition to Nebraska Tobacco Settlement Biomedical Research Development Funds. This is manuscript \#09 from the UNMC CITN. We thank Dr. Carolyn Keierleber and Denise Daggett for safety advice, Drs. Elizabeth Ford and Karen Clingerman for veterinary care (The Scripps Research Institutes); and Katy Emanuel for technical assistance, Robin Taylor for proofreading and figure graphics and design, and Dr. Gurudutt Pendyala for critically reading the manuscript (University of Nebraska Medical Center).

\section{Author details}

'Department of Pharmacology and Experimental Neuroscience, University of Nebraska Medical Center, Omaha, NE 68198, USA. ²Department of Immunology and Microbial Science, The Scripps Research Institute, La Jolla, CA 92037, USA. ${ }^{3}$ Molecular and Integrative Neuroscience Department, The Scripps Research Institute, La Jolla, CA 92037, USA.

\section{Authors' contributions}

SY carried out the in vitro experiments, helped design experiments and wrote the first draft of the manuscript. ADC assisted in the in vitro studies. CF performed work with the nonhuman primates. HF performed nonhuman primate work and designed experiments. All authors read and approved the final version of the manuscript.

\section{Competing interests}

The authors declare that they have no competing interests.

Received: 14 October 2010 Accepted: 21 July 2011

Published: 21 July 2011

\section{References}

1. Dauer W, Przedborski S: Parkinson's disease: mechanisms and models. Neuron 2003, 39:889-909.

2. Rodriguez-Oroz MC, Jahanshahi M, Krack P, Litvan I, Macias R, Bezard E, Obeso JA: Initial clinical manifestations of Parkinson's disease: features and pathophysiological mechanisms. Lancet Neurol 2009, 8:1128-1139.

3. Langston JW: The Parkinson's complex: parkinsonism is just the tip of the iceberg. Ann Neurol 2006, 59:591-596.

4. Bernheimer H, Birkmayer W, Hornykiewicz O, Jellinger K, Seitelberger F: Brain dopamine and the syndromes of Parkinson and Huntington. Clinical, morphological and neurochemical correlations. J Neurol Sci 1973, 20:415-455.

5. Marklund P, Larsson A, Elgh E, Linder J, Riklund KA, Forsgren L, Nyberg L: Temporal dynamics of basal ganglia under-recruitment in Parkinson's disease: transient caudate abnormalities during updating of working memory. Brain 2009, 132:336-346.

6. Dirnberger $\mathrm{G}$, Frith $\mathrm{CD}$, Jahanshahi M: Executive dysfunction in Parkinson's disease is associated with altered pallidal-frontal processing. Neuroimage 2005, 25:588-599.

7. Owen AM, Doyon J, Dagher A, Sadikot A, Evans AC: Abnormal basal ganglia outflow in Parkinson's disease identified with PET. Implications for higher cortical functions. Brain 1998, 121(Pt 5):949-965.

8. Dagher A, Owen AM, Boecker H, Brooks DJ: The role of the striatum and hippocampus in planning: a PET activation study in Parkinson's disease. Brain 2001, 124:1020-1032.

9. Monchi O, Petrides M, Mejia-Constain B, Strafella AP: Cortical activity in Parkinson's disease during executive processing depends on striatal involvement. Brain 2007, 130:233-244.

10. Ferri $\mathrm{KF}$, Kroemer $\mathrm{G}$ : Organelle-specific initiation of cell death pathways. Nat Cell Biol 2001, 3:E255-263.

11. Artal-Sanz M, Tavernarakis N: Proteolytic mechanisms in necrotic cell death and neurodegeneration. FEBS Lett 2005, 579:3287-3296.
12. Pope A, Nixon RA: Proteases of human brain. Neurochem Res 1984, 9:291-323.

13. Chu Y, Dodiya H, Aebischer P, Olanow CW, Kordower JH: Alterations in lysosomal and proteasomal markers in Parkinson's disease: relationship to alpha-synuclein inclusions. Neurobiol Dis 2009, 35:385-398.

14. Benes P, Vetvicka V, Fusek M: Cathepsin D-many functions of one aspartic protease. Crit Rev Oncol Hematol 2008, 68:12-28,

15. Cataldo AM, Nixon RA: Enzymatically active lysosomal proteases are associated with amyloid deposits in Alzheimer brain. Proc Natl Acad Sci USA 1990, 87:3861-3865.

16. Adamec E, Mohan PS, Cataldo AM, Vonsattel JP, Nixon RA: Up-regulation of the lysosomal system in experimental models of neuronal injury: implications for Alzheimer's disease. Neuroscience 2000, 100:663-675.

17. Kurlan R, Kim MH, Gash DM: Oral levodopa dose-response study in MPTPinduced hemiparkinsonian monkeys: assessment with a new rating scale for monkey parkinsonism. Mov Disord 1991, 6:111-118.

18. Imbert C, Bezard E, Guitraud S, Boraud T, Gross CE: Comparison of eight clinical rating scales used for the assessment of MPTP-induced parkinsonism in the Macaque monkey. J Neurosci Methods 2000, 96:71-76.

19. Nixon RA, Marotta CA: Degradation of neurofilament proteins by purified human brain cathepsin D. J Neurochem 1984, 43:507-516.

20. Decamp E, Schneider JS: Attention and executive function deficits in chronic low-dose MPTP-treated non-human primates. Eur J Neurosci 2004, 20:1371-1378.

21. Schneider JS, Kovelowski CJ: Chronic exposure to low doses of MPTP. I. Cognitive deficits in motor asymptomatic monkeys. Brain Res 1990, 519:122-128.

22. Roberg K, Ollinger $\mathrm{K}$ : Oxidative stress causes relocation of the lysosomal enzyme cathepsin $D$ with ensuing apoptosis in neonatal rat cardiomyocytes. Am J Pathol 1998, 152:1151-1156.

23. Roberg K, Johansson U, Ollinger K: Lysosomal release of cathepsin D precedes relocation of cytochrome $\mathrm{c}$ and loss of mitochondrial transmembrane potential during apoptosis induced by oxidative stress. Free Radic Biol Med 1999, 27:1228-1237.

24. Kagedal K, Johansson U, Ollinger K: The lysosomal protease cathepsin D mediates apoptosis induced by oxidative stress. FASEB J 2001, 15:1592-1594.

25. Ollinger K, Brunk UT: Cellular injury induced by oxidative stress is mediated through lysosomal damage. Free Radic Biol Med 1995, 19:565-574.

26. Miura Y, Sakurai Y, Hayakawa M, Shimada Y, Zempel H, Sato Y, Hisanaga S, Endo T: Translocation of lysosomal cathepsin D caused by oxidative stress or proteasome inhibition in primary cultured neurons and astrocytes. Biol Pharm Bull 33:22-28.

27. Jenner P: Oxidative stress in Parkinson's disease. Ann Neurol 2003, 53(Suppl 3):S26-36, discussion S36-28.

28. Guicciardi ME, Leist M, Gores GJ: Lysosomes in cell death. Oncogene 2004, 23:2881-2890.

29. Oberle C, Huai J, Reinheckel T, Tacke M, Rassner M, Ekert PG, Buellesbach J, Borner C: Lysosomal membrane permeabilization and cathepsin release is a Bax/Bak-dependent, amplifying event of apoptosis in fibroblasts and monocytes. Cell Death Differ 2010, 17:1167-1178.

30. Tatton WG, Chalmers-Redman R, Brown D, Tatton N: Apoptosis in Parkinson's disease: signals for neuronal degradation. Ann Neurol 2003, 53(Suppl 3):S61-70, discussion S70-62.

31. Briozzo P, Morisset M, Capony F, Rougeot C, Rochefort $H$ : In vitro degradation of extracellular matrix with Mr 52,000 cathepsin D secreted by breast cancer cells. Cancer Res 1988, 48:3688-3692.

32. Beaujouin M, Liaudet-Coopman E: Cathepsin D overexpressed by cancer cells can enhance apoptosis-dependent chemo-sensitivity independently of its catalytic activity. Adv Exp Med Biol 2008, 617:453-461.

33. Beaujouin M, Prebois C, Deroca D, Laurent-Matha V, Masson O, Pattingre S, Coopman P, Bettache N, Grossfield J, Hollingsworth RE, et al: Pro-cathepsin $D$ interacts with the extracellular domain of the beta chain of LRP1 and promotes LRP1-dependent fibroblast outgrowth. J Cell Sci 2010, 123:3336-3346

34. Ceccariglia S, D'Altocolle A, Del Fa A, Pizzolante F, Caccia E, Michetti F, Gangitano C: Cathepsin D plays a crucial role in the trimethyltin-induced hippocampal neurodegeneration process. Neuroscience 2011, 174:160-170.

35. Vitner EB, Dekel H, Zigdon H, Shachar T, Farfel-Becker T, Eilam R, Karlsson S, Futerman AH: Altered expression and distribution of cathepsins in 
neuronopathic forms of Gaucher disease and in other sphingolipidoses. Hum Mol Genet 2010, 19:3583-3590.

36. Umeda T, Tomiyama T, Sakama N, Tanaka S, Lambert MP, Klein WL, Mori H: Intraneuronal amyloid beta oligomers cause cell death via endoplasmic reticulum stress, endosomal/lysosomal leakage, and mitochondrial dysfunction in vivo. J Neurosci Res 2011, 89:1031-1042.

37. Boya P, Kroemer G: Lysosomal membrane permeabilization in cell death. Oncogene 2008, 27:6434-6451.

38. Sevlever $D$, Jiang $P$, Yen $S H$ : Cathepsin $D$ is the main lysosomal enzyme involved in the degradation of alpha-synuclein and generation of its carboxy-terminally truncated species. Biochemistry 2008, 47:9678-9687.

39. Katner SN, Flynn CT, Von Huben SN, Kirsten AJ, Davis SA, Lay CC, Cole M, Roberts AJ, Fox HS, Taffe MA: Controlled and behaviorally relevant levels of oral ethanol intake in rhesus macaques using a flavorant-fade procedure. Alcohol Clin Exp Res 2004, 28:873-883.

40. Marcondes MC, Burdo TH, Sopper S, Huitron-Resendiz S, Lanigan C, Watry D, Flynn C, Zandonatti M, Fox HS: Enrichment and persistence of virus-specific CTL in the brain of simian immunodeficiency virus-infected monkeys is associated with a unique cytokine environment. J Immunol 2007, 178:5812-5819.

doi:10.1186/1750-1326-6-52

Cite this article as: Yelamanchili et al:: Upregulation of cathepsin D in

the caudate nucleus of primates with experimental parkinsonism.

Molecular Neurodegeneration 2011 6:52.

\section{Submit your next manuscript to BioMed Central} and take full advantage of:

- Convenient online submission

- Thorough peer review

- No space constraints or color figure charges

- Immediate publication on acceptance

- Inclusion in PubMed, CAS, Scopus and Google Scholar

- Research which is freely available for redistribution

Submit your manuscript at www.biomedcentral.com/submit
C Biomed Central 